\title{
Quantitative Description of Vertical Organic Matter Distribution in Real Soil Profiles by Means a Simple Continuous Model
}

\author{
Sergey I. Bartseva, ${ }^{\mathrm{a}, \mathrm{b}}$, Aleksei A. Pochekutov ${ }^{\mathrm{a}, *}$ \\ ${ }^{a}$ Institute of Biophysics SB RAS, Federal Research Center "Krasnoyarsk Science Center SB \\ RAS", Krasnoyarsk, Russia \\ ${ }^{b}$ Institute of Fundamental Biology and Biotechnology of Siberian Federal University, \\ Krasnoyarsk, Russia
}

\begin{abstract}
Previously we have proposed a continuous model of soil organic matter (SOM) transformation which was based on describing only the most general notions of this process - a gradual increase in SOM stability toward transformation, occurring concurrently with partial decomposition of SOM. The model provided qualitative description of vertical SOM distributions in different soils. In the present study this model has been modified to make the description more realistic. The study demonstrates quantitative correspondence between the calculated and averaged observed vertical distributions of SOM for different biomes. Keywords: soil organic matter, continuous model of soil, soil profile, vertical soil organic matter distribution
\end{abstract}

\section{1. Introduction}

2 In a previous study, we proposed a simple continuous model of SOM transfor3 mation and decomposition (Bartsev and Pochekutov, 2015). That model was

4 based on the most general notions of these processes: a gradual increase in 5 the stability of SOM toward transformation, which occurs simultaneously with

\footnotetext{
${ }^{*}$ Corresponding author

Email addresses: bartsev@yandex.ru (Sergey I. Bartsev), a-a-po@mail.ru (Aleksei A. Pochekutov)
}

Preprint submitted to Ecological Modelling 
decomposition of some part of SOM. The continuous scale of stability of the matter used in the basic model was the rate of further transformation of SOM into more stable forms. Then, we proposed a modified model, which established a one-to-one correspondence between the stationary SOM distributions along the transformation rate and along the depth in soil profile. For that model, we demonstrated qualitative correspondence of the patterns of SOM vertical distribution curves to those for various types of real soils (Bartsev and Pochekutov, 2016).

Although derivation of model equations in the previous papers (Bartsev and Pochekutov, 2015, 2016) was described in terms of the classical theory of humification (Essington, 2004), the model can be used within the framework of both this theory and any other SOM transformation concepts that suggest a gradual increase in SOM stability toward transformation and decomposition. These are the concepts suggesting, e.g., an increase in SOM stability caused by an increase in its inaccessibility and protection against decomposers (v. Lützov et al., 2006) or even by an increase in the proportion of stable compounds in the plant litter due to more rapid mineralization of readily mineralized substances (Berg and McClaughretv, 2008). The reason why this model is so universal is that it has been constructed using a very simple approach, which involves a phenomenological representation of the most general notions about the nature and direction of SOM transformation process, providing no details or internal mechanisms of these processes, which would connect the model to certain theoretical notions of organic matter transformation in soil.

\section{The model equations and their new modification}

\subsection{The transformation equations}

The basic equation of the model (Bartsev and Pochekutov, 2015):

$$
\frac{\partial C(h, t)}{\partial t}-\frac{\partial}{\partial h}\left(h^{2} C(h, t)\right)=-k(h) C(h, t)+D(h, t)
$$

describes SOM transformation as movement of the matter along a continuous scale representing the degrees of stability of the matter toward further trans- 
formation (including decomposition). The rate of SOM transformation into a more stable form is used as this scale, $h$. In terms of the classical theory of humification, $h$ means the rate of humification of the matter. $C(h, t)$ is SOM distribution along scale $h$, changing over time; $k(h)$ is coefficient of mineralization rate, assigned in the existing versions of the model from the empirical function $k(h)=b h^{p}$, where $b$ and $p$ are adjustable parameters (interpreted elsewhere (Bartsev and Pochekutov, 2016)). Plant litter is described by its input rate $D(h, t)$, which is defined in the model from an approximate equation

$$
D(h)=\sum_{i} D_{0 i} \delta\left(h-h_{0 i}\right)
$$

where $\delta$ is the Dirac delta function, and index $i$ numbers plant litter components characterized by their annual average amount $D_{0 i}$ and initial transformation rate $h_{0 i}$.

When plant litter input is defined by (2), equation (11) can be written for $C_{i}(h, t)$ - the distribution of transformation products of each litter component. Then, the distribution of the total SOM is expressed as $C(h, t)=\sum_{i} C_{i}(h, t)$.

In the stationary case, solution of equation (11) written for $C_{i}(h, t)$ has the following form:

$$
\bar{C}_{i}(h)=\frac{D_{0 i}}{h^{2}} \exp \left(\frac{b}{p-1}\left(h^{p-1}-h_{0 i}^{p-1}\right)\right)\left(1-\theta\left(h-h_{0 i}\right)\right),
$$

where $\theta$ is the Heaviside step function.

In this study, in order to bring the model concepts closer to the notions of the nature of soil processes, we propose a modified model, which assumes that not all matter in the soil simultaneously undergoes transformation.

In any stage of transformation of the matter, only some part of this matter undergoes transformation at any given time. If reactions of both decomposition and transition to a more stable form are described by the laws of chemical kinetics, at any moment there is a part of the matter that has entered into the reaction and a part of the matter that has not. Then, equation (11) only describes the dynamics of the part of the matter that undergoes transformation at a given time. Moreover, for reasons described in a review by v. Lützov et al. 
(2006), some of the SOM may become inaccessible to the effects of biological or chemical factors causing transformation of the matter.

Without going into great detail, we can describe the total result of the operation of these mechanisms in a generalized way as follows. The part of the SOM that is undergoing transformation at a given time will be called active SOM, and its distribution will be denoted by $c(h, t)$. The other part of the SOM, which is not undergoing transformation at the same time, will be called inactive SOM, and its distribution will be denoted by $s(h, t)$. The distribution of the total SOM will be expressed as $C(h, t)=c(h, t)+s(h, t)$. Assuming that the rate of transition of the matter from the active state to the inactive one can be expressed as $\beta(h) c(h, t)$ and the rate of transition of the matter from the inactive state to the active one as $\alpha(h) s(h, t)$, we obtain a system of equations describing the total SOM dynamics in both forms:

$$
\begin{aligned}
\frac{\partial c(h, t)}{\partial t}-\frac{\partial}{\partial h}\left(h^{2} c(h, t)\right)=-b h^{p} c(h, t)+D(h, t) & -\beta(h) c(h, t)+\alpha(h) s(h, t) \\
\frac{\partial s(h, t)}{\partial t} & =\beta(h) c(h, t)-\alpha(h) s(h, t)
\end{aligned}
$$

In the stationary case, $\beta(h) \bar{c}(h)=\alpha(h) \bar{s}(h)$, and (4) will assume the form that fully coincides with the stationary form of equation (1), and, hence, the solution of this equation will have the form of (3). Then, the total stationary distribution of SOM will be

$$
\bar{C}(h)=\left(1+\frac{\beta(h)}{\alpha(h)}\right) \bar{c}(h) .
$$

Let us assume that $\beta(h)$ and $\alpha(h)$ can be approximated by the simplest linear functions, $\beta(h)=q h, \alpha(h)=r h$, where $q$ and $r$ are positive constants. Then, the distribution of the products of transformation of the $i$-th litter component will take the following form:

$$
\bar{C}_{i}(h)=\left(1+\frac{q}{r}\right) \frac{D_{0 i}}{h^{2}} \exp \left(\frac{b}{p-1}\left(h^{p-1}-h_{0 i}^{p-1}\right)\right)\left(1-\theta\left(h-h_{0 i}\right)\right) .
$$

In this modification of the model, only one new parameter, $q / r$, is introduced into the equations. This parameter denotes how many times the amount of inactive SOM is different from the amount of active SOM. 


\subsection{The vertical transport equations}

In order to establish one-to-one correspondence between the stationary SOM distributions along the transformation rate $h$ and along the depth $z$ in soil profile, one should assign $w(h) \equiv \mathrm{d} z / \mathrm{d} t$. The equation of relation between scales

$h$ and $z$ that we derived previously (Bartsev and Pochekutov, 2016, Eq.9), in the general case for the arbitrary $w(h)$ form, will be written as

$$
\frac{\mathrm{d} h}{\mathrm{~d} z}=-\frac{h^{2}}{w(h)} .
$$

The stationary distribution of SOM along the scale $z, \overline{\mathcal{C}}(z)$, will be related to the stationary distribution of SOM along the scale $h, \bar{C}(h)$, by the equation

$$
\overline{\mathcal{C}}(z)=J \cdot \bar{C}(h(z))
$$

where $J \equiv-\mathrm{d} h / \mathrm{d} z$ is transition Jacobian from scale $h$ to scale $z$ (Bartsev and Pochekutov, 2016).

While previously (Bartsev and Pochekutov, 2016) we assumed $w(h)=a h$, we now assume

$$
w(h)=a h+A,
$$

where $a$ and $A$ are nonnegative constants. Thus, for any substance, its vertical transport velocity, $w$, consists of two components: one component is determined by the stability of the substance and the other is the same for all substances in the soil. This also brings model concepts closer to processes in real soils. Summand $A$ is added to take into account possible vertical transport factors that affect particles of the matter irrespective of its degree of transformation, such as transport of particles with the liquid when large amounts of water percolate through the soil.

By solving the differential equation (8) taking into account (10) and initial condition $h(z=0)=h_{0 i}$, we obtain a new expression of the relation between scales $z$ and $h$ :

$$
z=a \log \left(\frac{h_{0 i}}{h}\right)+A\left(\frac{1}{h}-\frac{1}{h_{0 i}}\right) .
$$

8 The function $h(z)$ necessary for further computations can only be obtained from this by numerically solving the transcendental equation (11) relative to $h$. For 
each plant litter component that differs from other components in the $h_{0 i}$ value, function $h(z)$ must be calculated individually, using this very value of $h_{0 i}$.

As follows from (8) and (10), Jacobian $J$ in equation (91) assumes the form $\left(h^{2}(z)\right) /(a h(z)+A)$. Then equation (9), taking into account (7) for products of transformation of the $i$-th component of plant litter will be written as

$$
\overline{\mathcal{C}}_{i}(z)=\frac{\left(1+\frac{q}{r}\right) D_{0 i}}{a h(z)+A} \exp \left(\frac{b}{p-1}\left(h^{p-1}(z)-h_{0 i}^{p-1}\right)\right) \theta(z) .
$$

Equation (12) holds for products of transformation of plant litter components falling onto soil surface such as aboveground parts of plants. To make an accurate description of the root litter, one should take into account that roots and, hence, root litter are distributed over depth along the soil profile. To take into account the depth-distributed input of the root litter, one must know its distribution function, $\mathcal{D}_{R}(z, h)$. In the simple case, if all root litter is described by one value of $h_{0}$, this will be function $\mathcal{D}_{R}(z)$. Then, the amount of the root litter input in a depth micro-range between $z_{0}$ to $z_{0}+\mathrm{d} z$ will be equal to $\mathcal{D}_{R}\left(z_{0}\right) \mathrm{d} z$. The stationary distribution of the transformation products of this litter portion will be described by the equation with $\left(z-z_{0}\right)$ substituted for $z$ and $\mathcal{D}_{R}\left(z_{0}\right) \mathrm{d} z$ substituted for $D_{0 i}$. Then, the stationary distribution of the products of transformation of the total root litter is described by equation

$$
\overline{\mathcal{C}}_{R}(z)=\int_{0}^{z_{m}} \frac{\left(1+\frac{q}{r}\right) \mathcal{D}_{R}(\zeta)}{a h(z-\zeta)+A} \exp \left(\frac{b\left(h^{p-1}(z-\zeta)-h_{0 i}^{p-1}\right)}{p-1}\right) \theta(z-\zeta) \mathrm{d} \zeta
$$

where $z_{m}$ is the maximum depth where roots are found.

We use equations (12) and (13) in the section below to calculate the SOM vertical distributions compared to the field data.

\section{Fitting of model calculated SOM distributions}

The model was tested by comparing model calculations to the SOM vertical distributions observed in nature.

As field data on the SOM (in carbon units) vertical distribution in real soils, we used the data averaged for biomes on organic carbon in soils at dif- 
ferent depths presented in a study by Jobbágy and Jackson (2000). The authors of that study used three soil databases: the National Soil Characterization Database (NSCD) (USDA, 1994), the World Inventory of Soil Emission Potential Database (WISE) (Baties and Bridges, 1994; Baties, 1995), and the database from the Canadian Forest Service (Siltanen et al., 1997).

Based on the values of total organic carbon $S$ in the soil layer and its percentage $P_{j}$ in the depth range between $z_{j-1}$ and $z_{j}$ (Jobbágv and Jackson, 2000 , Table 3 and 4), we calculated approximate values of the function of the observed SOM vertical distribution:

$$
\mathcal{C}_{n a t}\left(z_{j-1}+\frac{z_{j}-z_{j-1}}{2}\right) \approx \frac{0.01 \cdot P_{j} \cdot S}{z_{j}-z_{j-1}}
$$

For three biomes - boreal forest, temperate grassland, and tropical evergreen forest, we calculated the SOM distributions $\overline{\mathcal{C}}_{i}(z)$ using equations (12) and (13) and fitted the values (individually for each biome) of parameters $b, p, a, A$, $q / r$ in order to minimize the discrepancy between $\mathcal{C}_{n a t}(z)$ and $\overline{\mathcal{C}}(z)=\sum_{i} \overline{\mathcal{C}}_{i}(z)$.

In the calculations, plant litter was divided into three components leaf, root, and wood litter, each characterized by its own values of $D_{0 i}$ (in $\left.\mathrm{kgC} \cdot \mathrm{m}^{-2} \mathrm{yr}^{-1}\right)$ and $h_{0 i}\left(\right.$ in $\left.\mathrm{yr}^{-1}\right)$. Hence, respectively, $i=\{L, R, W\}$.

The data on the annual average amounts of litter components in the biomes studied here were taken from (Rodin and Bazilevich, 1967) and multiplied by coefficient 0.5 to approximately convert them into carbon units.

Calculations of $h_{0 i}$ values were based on the literature data on initial rates of litter mineralization, $k_{0 i}$, using the relation between these values accepted in the model: $h_{0 i}=\left(k_{0 i} / b\right)^{1 / p}$.

For the boreal forest, we used the values of $k_{0 i}$ given by (Vedrova, 1995, 2005), which were averaged for all the species and all the taiga types considered. For the temperate grassland, we used averaged values of $k_{0 i}$ of roots and aboveground parts of herbaceous plants measured in temperate latitudes taken from (Bontti et al., 2009). For the tropical forest, the values of $k_{0 i}$ for the wood litter were taken from (Chambers et al., 2000) and for the root and leaf litter from (Gholz et al., 2000). 
Table 1: Litter characteristics used in the calculations

\begin{tabular}{lcccccc}
\hline & \multicolumn{3}{c}{ Leaf litter } & \multicolumn{2}{c}{ Root litter } & \multicolumn{2}{c}{ Wood litter } \\
& $D_{0 L}$ & $k_{0 L}$ & $D_{0 R}$ & $k_{0 R}$ & $D_{0 W}$ & $k_{0 W}$ \\
\hline Boreal forest & 0.121 & 0.137 & 0.028 & 0.072 & 0.079 & 0.023 \\
Temperate grassland & 0.19 & 0.28 & 0.29 & 0.31 & - & - \\
Tropical forest & 0.798 & 1.46 & 0.083 & 0.739 & 0.495 & 0.17 \\
\hline
\end{tabular}

Table 2: The fitted values of model parameters

\begin{tabular}{lccccc}
\hline & $b$ & $p$ & $a$ & $A$ & $q / r$ \\
\hline Boreal forest & 0.181608 & 0.420912 & 0.678584 & 0.000109 & 0.977158 \\
Temperate grassland & 0.638244 & 0.957071 & 0.016981 & 0.002430 & 0.260165 \\
Tropical forest & 0.761897 & 0.980069 & 0.0 & 0.010613 & 0.130199 \\
\hline
\end{tabular}

The vertical distributions of the root litter in calculations using equation (13) were obtained by linear interpolation of the literature data. The amount of the root litter input $L_{j}$ within the depth range between $z_{j-1}$ and $z_{j}$ was evaluated as a portion of the root biomass within this range (from (Jobbágv and Jackson 2000. Table 4)) multiplied by the total root litter (from (Rodin and Bazilevich, 1967)). For each $j$-th depth rang, the function $\mathcal{D}_{R}(z)$ was interpolated as

$$
\mathcal{D}_{R j}(z)=g_{j} z+G_{j} \quad \text { if } \quad z_{j-1}>z \geq z_{j}
$$

where $j=1,2,3,4,5, z_{\{0,1,2,3,4,5\}}=\{0,0.2,0.4,0.6,0.8,1\} \mathrm{m}$, and constants $g_{j}$ and $G_{j}$ were set so that $\int_{z_{j-1}}^{z_{j}} \mathcal{D}_{R j}(z) \mathrm{d} z=L_{j}$, and $g_{j} z_{j}+G_{j}=g_{j+1} z_{j}+G_{j+1}$ for $j=1,2,3,4$ and $g_{5} z_{5}+G_{5}=0$.

The initial data used in calculations are listed in Table 1 the fitted values of model parameters are given in Table 2 and the corresponding distribution curves are shown in Figure 1

Data in Table 2 suggest certain patterns of change in model parameters. For instance, values of parameters $b$ and $p$ increase with the transition from colder to warmer climate zones. This can be interpreted as an increase in the proportion of the mineralized SOM, which is particularly characteristic of unstable substances 


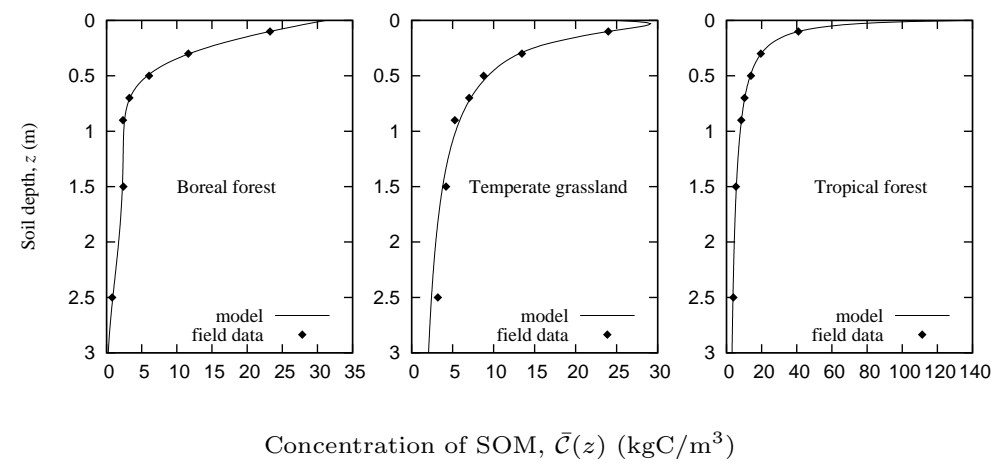

Figure 1: Model curves of the SOM vertical distribution compared to field data

in early phases of transformation, occurring with the transition from colder to warmer climate. This, in turn, is consistent with the general concept of an increase in the rates of biological processes. Another trend is a monotonic change of the values of $a, A$, and $q / r$ with the transition from boreal forest to temperate grassland and, then, to tropical forest. Also interestingly, the value of $a$ for the tropical forest is minimal and equal to 0 while the value of $A$ there is 1-2 orders of magnitude higher than in the other biomes. A possible interpretation of this may be that the process of SOM vertical transport is dominated by factors that are not related to the degree of transformation and stability of the matter. Percolation of large amounts of precipitated water through the soils in tropical rainforests (Zech and Hintermaier-Erhard, 2007) can act as this factor.

\section{Discussion}

Other authors (Nakane and Shinozaki, 1978; Bosatta and Ågren, 1996) have used continuous models of SOM transformation to calculate the SOM vertical distributions. In those studies, however, model calculations were compared with the field data on the distribution of organic matter in the soils of individual ecosystems. In this study, we compare model calculations to the observed SOM distributions averaged for biomes. Comparison of model calculations with the averaged data seems appropriate for the proposed model, as this model describes 
SOM transformation processes in the most general terms, and averaging of the field data reduces the differences between individual ecosystems. If these differences were taken into account in the model, it would probably become more intricate.

The model proposed here compares favorably with the model described by (Bosatta and Åren, 1996) and other models of the Q-model family (Ågren and Bosatta, 1998; Bosatta and Ågren, 2003), as the basic notions about SOM transformation process included in the model are simple, even elementary, and only reflect the general trend of the process. Therefore, equations of our model are simpler and more transparent, and the model contains just a minimal set of fitting parameters.

The model described in a study by Nakane and Shinozaki (1978) is also very simple and uses simple empirical functions to describe the processes, providing rather good correspondence between calculations and the field data. However, that model describes the increase in SOM stability and its vertical transport as a single whole, related by the generality and uniqueness of the scale - depth $z$. One of the advantages of our model over the model proposed by Nakane and Shinozaki is that our model equation has been obtained from explicit assumptions about the general patterns of SOM transformation and mineralization processes. Moreover, in our model, the increase in stability (Section 2.1) is described separately from the vertical transport (Section 2.2), and, thus, as the model develops, the notions about either of these processes may be modified and specified independently of each other. This will provide more flexibility in the possible further development of the model, bringing it closer to the description of real processes.

\section{Conclusion}

In this study, we demonstrated quantitative correspondence between the SOM vertical distributions calculated using the proposed model and the vertical distributions of organic matter averaged for biomes that are observed in real 
soils. That was achieved by using rather simple mathematical tools and a small number of parameters. Thus, the proposed model is a convenient instrument for the initial coordination of field dataset components providing self-contained description of SOM dynamics.

\section{Acknowledgements}

This study was supported by the Russian Science Foundation (project No. 16-14-10115).

\section{References}

Ågren, G.I., Bosatta, E., 1998. Theoretical Ecosystem Ecology: Understanding Element Cycles. Cambridge University Press, Cambridge.

Bartsev, S.I., Pochekutov, A.A., 2015. A continual model of soil organic matter transformations based on a scale of transformation rate. Ecological Modelling $302,25-28$.

Bartsev, S.I., Pochekutov, A.A., 2016. The vertical distribution of soil organic matter predicted by a simple continuous model of soil organic matter transformations. Ecological Modelling 328, 95-98.

Batjes, N.H., 1995. A homogenized soil data file for global environmental research: a subset of FAO, ISRIC and NRCS profiles (Version 1.0). Working Paper and Preprint 95/10. ISRIC, Wageningen, The Netherlands.

Batjes, N.H., Bridges, E.M., 1994. Potential emissions of radiatively active gases from soil to atmosphere with special reference to methane: development of a global database (WISE). Journal of Geophysical Research 99, 16479-16489.

Berg, B., McClaughrety, C., 2008. Plant Litter: Decomposition, Humus Formation, Carbon Sequestration. Springer-Verlag, Berlin, Heidelberg. 
Bontti, E.E., Decant, J.P., Munson, S.M., Gathany, M.A., Przeszlowska, A., Haddix, M.L., Owens, S., Burke, I.C., Parton, W.J., Harmon, M.E., 2009. Litter decomposition in grasslands of Central North America (US Great Plains). Global Change Biology 15, 1356-1363.

Bosatta, E., Ågren, G.I., 1996. Theoretical analyses of carbon and nutrient dynamics in soil profiles. Soil Biology and Biochemistry 28, 1523-1531.

Bosatta, E., Ågren, G.I., 2003. Exact solutions to the continuous-quality equation for soil organic matter turnover. Journal of Theoretical Biology 224, $97-105$.

Chambers, J.Q., Higuchi, N., Schimel, J.P., Ferreira, L.V., Melack, J.M., 2000. Decomposition and carbon cycling of dead trees in tropical forests of the central Amazon. Oecologia 122, 380-388.

Essington, M.E., 2004. Soil and water chemistry: an integrative approach. CRC Press. pp. $157-163$.

Gholz, H.L., Wedin, D.A., Smitherman, S.M., Harmon, M.E., Parton, W.J., 2000. Long-term dynamics of pine and hardwood litter in contrasting environments: toward a global model of decomposition. Global Change Biology $6,751-765$.

Jobbágy, E.G., Jackson, R.B., 2000. The vertical distribution of soil organic carbon and its relation to climate and vegetation. Ecological Applications 10, 423-436.

v. Lützov, M., Kögel-Knabner, I., Ekschmitt, K., Mantzer, E., Guggenberger, G., Marschner, B., Flessa, H., 2006. Stabilization of organic matter in temperate soils: mechanisms and their relevance under different soil conditions — a review. European Journal of Soil Science 57, 426-445.

Nakane, K., Shinozaki, K., 1978. The vertical distribution of soil organic carbon and its relation to climate and vegetation. Japanese Journal of Ecology 28, 111-122. 
Rodin, L.E., Bazilevich, N., 1967. Production and Mineral Cycling in Terrestrial Vegetation. Oliver and Boyd, Edinburgh.

Siltanen, R.M., Apps, M.J., Zoltai, S.C., Mair, R.M., Strong, W.L., 1997. A soil profile and organic carbon data base for Canadian forest and tundra mineral soils. Canadian Forestry Service, Edmonton, Canada.

USDA, 1994. National Soil Characterization Data. Soil Survey Laboratory, National Soil Survey Center, Soil Conservation Service, Lincoln, Nebraska, USA.

Vedrova, E.F., 1995. Carbon pools and fluxes of 25-year old coniferous and deciduous stands in Middle Siberia. Water, Air and Soil Pollution 82, 239246.

Vedrova, E.F., 2005. Biochemistry of carbon and nitrogen in the siberian afforestation experiment, in: Binkley, D., Menyailo, O. (Eds.), Tree Species Effects on Soils: Implications for Global Change. Kluwer Academic Publishers. NATO Science Series, pp. 281-290.

Zech, W., Hintermaier-Erhard, G., 2007. Soils of the World. Springer-Verlag, Heidelberg, Berlin. 